criteria were age $\geq 35$ years, COPD diagnosis, forced expiratory volume in 1 second/forced vital capacity (FEV $1 / \mathrm{FVC}$ ) $<0.7$, linkage to HES, and continuous GP registration for 12 months pre-index and 6 months post-index. Patients were new users of MITT or FF/UMEC/VI at index. Inverse probability of treatment weighting (IPTW) was used to balance baseline characteristics including sociodemographics, $\mathrm{FEV}_{1} \%$ predicted, symptom scores, prior exacerbations, prior respiratory therapy and comorbidities. Adherence was measured using proportion of days covered (PDC) by days' supply of FF/UMEC/VI or MITT prescriptions over 6,12, and 18 months post-index. PDC calculations did not include potential stockpiling and MITT patients were required to have supply of all components for days to be considered covered. Analyses included both proportion of adherent patients (PDC $\geq 0.80$ ) and mean PDC.

Results In total, 1,319 FF/UMEC/VI and 4,092 MITT users met the inclusion criteria. IPTW provided good balance of baseline characteristics demonstrated by standardised mean difference $<10 \%$ for all covariates. Patients with COPD initiating FF/UMEC/VI had significantly higher adherence than MITT users over 6, 12 and 18 months, using both categorical (PDC $\geq 0.80$ ) and continuous (mean PDC) adherence measures (Results presented in table 1); $\mathrm{p} \leq 0.001$ for all comparisons.

Conclusion In the UK, patients with COPD initiating FF/ UMEC/VI have significantly better adherence compared with patients initiating MITT.

\section{S28 HOME HUMIDIFIED HIGH-FLOW THERAPY FOLLOWING SEVERE EXACERBATION OF COPD: A MIXED-METHODS FEASIBILITY RANDOMISED CONTROL TRIAL}

${ }^{1}$ RF D'Cruz, ${ }^{1} A$ Rossel, 'ES Suh, ' $\mathrm{G}$ Kaltsakas, ${ }^{1} \mathrm{NM}$ Shah, ${ }^{2} \mathrm{~A}$ Douiri, ${ }^{3} \mathrm{~L}$ Rose, ${ }^{1} \mathrm{~PB}$ Murphy, ${ }^{1} \mathrm{~N}$ Hart. 'Lane Fox Clinical Respiratory Physiology Research Unit, Guy's and St Thomas' NHS Foundation Trust, London, UK; ${ }^{2}$ School of Population Health and Environmental Sciences, King's College London, London, UK; ${ }^{3}$ Florence Nightingale Faculty of Nursing, Midwifery and Palliative Care, King's College London, London, UK

\subsection{6/thorax-2021-BTSabstracts.34}

Introduction Patients recovering from severe acute exacerbations of COPD (AECOPD) have a 30-day readmission rate of

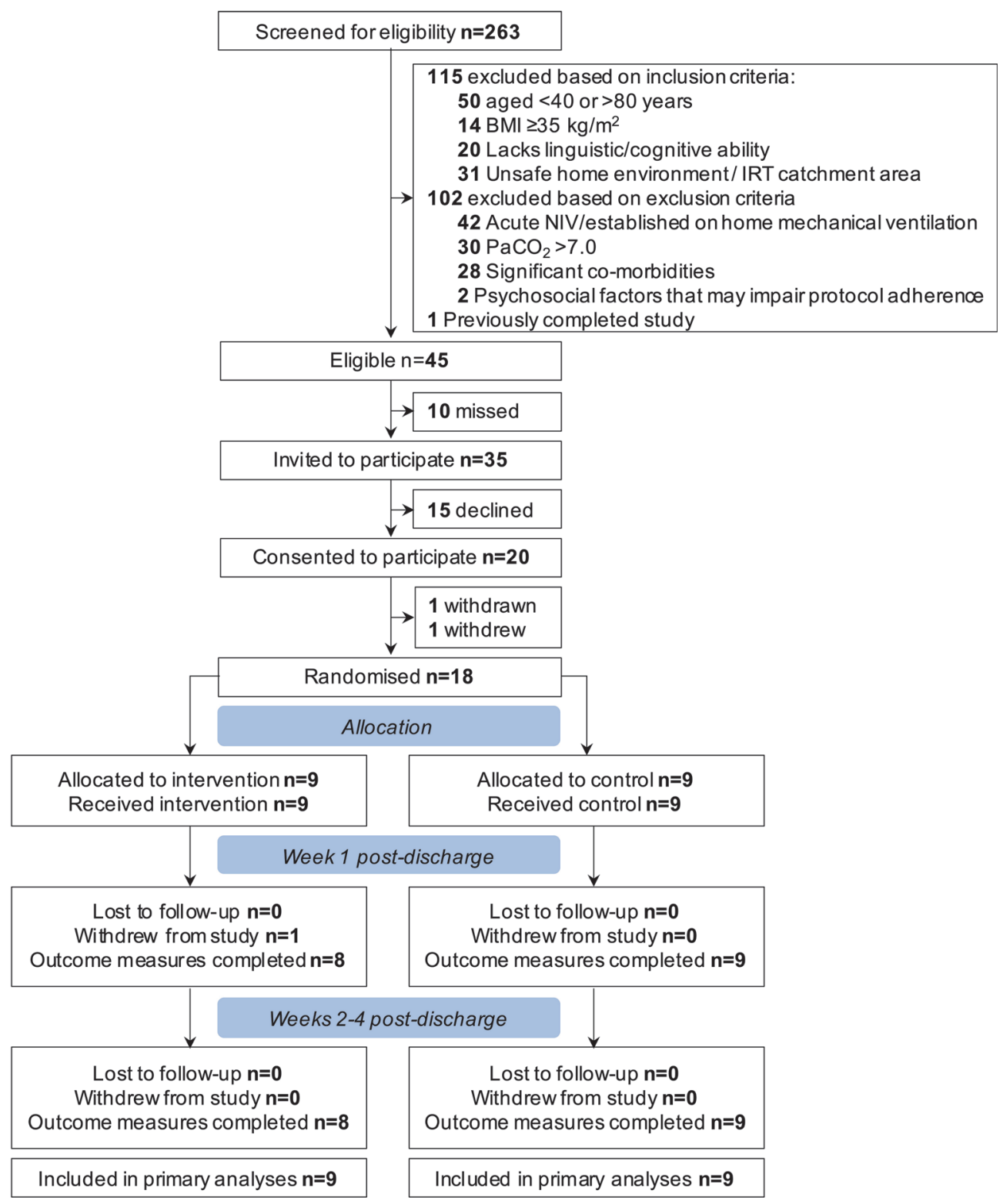

Abstract S28 Figure 1 Patient flow diagram 
25\%. High-flow therapy (HFT) improves mucociliary clearance, dead space washout and pulmonary mechanics in stable COPD. This study aimed to determine the feasibility of a Phase III randomised control trial (RCT) of home HFT to improve clinical, patient-reported and physiological outcomes following severe AECOPD.

Methods Mixed-methods feasibility RCT (quantitative primacy, concurrently embedded qualitative evaluation) (NCT03899558). Consecutive hospitalised AECOPD patients, aged $40-80, \quad \mathrm{BMI} \leq 35 \mathrm{~kg} / \mathrm{m}^{2}, \quad \mathrm{PaCO}_{2} \leq 7 \mathrm{kPa}$, not requiring acute or home mechanical ventilation were randomised to receive usual care or additional home HFT and received weekly home-based follow-up. Semi-structured interviews were performed in week 4. Feasibility outcomes were recruitment, protocol adherence, device acceptability. Progression criteria: $\geq 40 \%$ of eligible patients randomised, complete data in $>70 \%$, no device-related serious adverse events (SAE).

Results Feasibility: Between June 2019-March 2020, 263 patients screened, 45 eligible, 15 declined, 18 (40\%) randomised (figure 1). Mean $\pm \mathrm{SD} /$ median(IQR) age $69 \pm 5,44 \%$ female, BMI $22.5 \pm 5 \mathrm{~kg} / \mathrm{m}^{2}, \mathrm{FEV}_{1} 32 \pm 12 \%$. Discharge HFT settings: $37^{\circ} \mathrm{C}, 25-30 \mathrm{~L} / \mathrm{min}$. Adherence to assessments, questionnaires and parasternal EMG (EMG $\mathrm{Eara}) 100 \%$, spirometry $91 \%$, complete data in $83 \%$. By week 4, HFT use was 2.4 hours/day. There were no device-related SAE. Four themes relating to HFT acceptability were identified: technical, daily routine, impact on symptoms, sensory-affective influence. Facilitators to use included device simplicity and sputum clearance, barriers were warmup time and excessive flow/temperature. Exploratory: The HFT group had a 59\% risk of 30-day reexacerbation/readmission (OR $0.41, \quad 95 \%$ CI $\quad 0.05-3.31$ ). Breathlessness (mBorg) and neural respiratory drive index $\left(\mathrm{EMG}_{\mathrm{para} \% \max } \cdot \mathrm{bpm}\right.$ ) improved in the HFT group (admission to week 4 change $(\Delta)$ and repeated measures ANOVA significance: $\Delta-5(-6$ to -3$), \mathrm{p}<0.001$ and $\Delta-214 \pm 95 \% . \mathrm{bpm}, \mathrm{p}=0.02$, respectively), not controls $(\Delta-2 \pm 3, \mathrm{p}=0.25$ and $\Delta-207 \pm 299 \%$. $\mathrm{bpm}, \mathrm{p}=0.10$, respectively). Health status (COPD assessment test) improved in the HFT $(\Delta-12 \pm 5, \mathrm{p}<0.001)$ and control groups $(\Delta-12 \pm 7, p=0.01)$. Total sleep time fell in the HFT group $(\Delta-84 \pm 97 \mathrm{~min}, \mathrm{p}=0.02)$. There were no changes in physical activity.

Conclusions This study design was determined to be feasible, with all progression criteria met. A Phase III RCT is warranted to evaluate the effects of home high-flow therapy on 30-day re-exacerbation/readmission and patient-reported and physiological outcomes in this high-risk cohort.

\section{S29 PHYSICAL ACTIVITY AND SLEEP QUALITY AS RELATED TO PATIENT-REPORTED OUTCOMES AND PHYSIOLOGY DURING RECOVERY FROM SEVERE COPD EXACERBATION}

${ }^{1}$ RF D'Cruz, ${ }^{1}$ ES Suh, ${ }^{2} \mathrm{M}$ Patout, ${ }^{1} \mathrm{G}$ Kaltsakas, ${ }^{1} \mathrm{NM}$ Shah, ${ }^{3} \mathrm{R}$ Priori, ${ }^{4} \mathrm{~A}$ Douiri, ${ }^{5} \mathrm{~J}$ Moxham, ${ }^{1} \mathrm{~N}$ Hart, 'PB Murphy. 'Lane Fox Clinical Respiratory Physiology Research Unit, Guy's and St Thomas' NHS Foundation Trust, London, UK; ${ }^{2} A P-H P$, Groupe Hospitalier Universitaire APHP-Sorbonne Université, site Pitié-Salpêtrière, Service des Pathologies du Sommeil (Département R3S) and Sorbonne Université, INSERM, UMRS1158 Neurophysiologie Respiratoire Expérimentale et Clinique, Paris, France; ${ }^{3}$ Philips Research, Eindhoven, The Netherlands; ${ }^{4}$ School of Population Health and Environmental Sciences, King's College London, London, UK; ${ }^{5}$ Centre for Human and Applied Physiological Sciences, King's College London, London, UK

10.1136/thorax-2021-BTSabstracts.35
Introduction Physical activity (PA) and sleep quality are commonly impaired in COPD, are associated with increased exacerbation frequency, healthcare utilisation and death, and deteriorate during acute exacerbations (AECOPD). Their postdischarge trajectories following hospitalisation with AECOPD and associations with patient-reported and physiological outcomes have not been reported. This study aimed to evaluate (1) daily changes in PA and sleep, (2) influences of individual characteristics on PA and sleep and (3) relationships between $\mathrm{PA}$ and sleep and patient-reported and physiological outcomes following severe AECOPD.

Methods Prospective, single-centre observational study (NCT03443505,NCT01361451). Hospitalised AECOPD patients underwent wrist-worn actigraphy monitoring for 28 days post-discharge and were evaluated 1 - and 4 -weeks postdischarge.

Results Data from 1601 days and 1415 nights from 67 patients were analysed. Mean \pm SD/median(IQR) age $69 \pm 9$, $57 \%$ female, BMI 22.4(18.9-28.4) $\mathrm{kg} / \mathrm{m}^{2}, \mathrm{FEV}_{1}$ 27\%predicted, $24 \%$ readmitted within 28 days. Using repeated measures ANOVA, PA increased in the 4 weeks post-discharge $(F=8.47$, $\mathrm{p}<0.001)$ and was lower in those with $\mathrm{FEV}_{1}<30 \%$ predicted (figure 1a), and total sleep time fell $(F=2.70, \mathrm{p}=0.049)$. A circadian rhythm of PA was plotted using 2,898,935 30-second epochs (figure 1b). Linear mixed-model regression
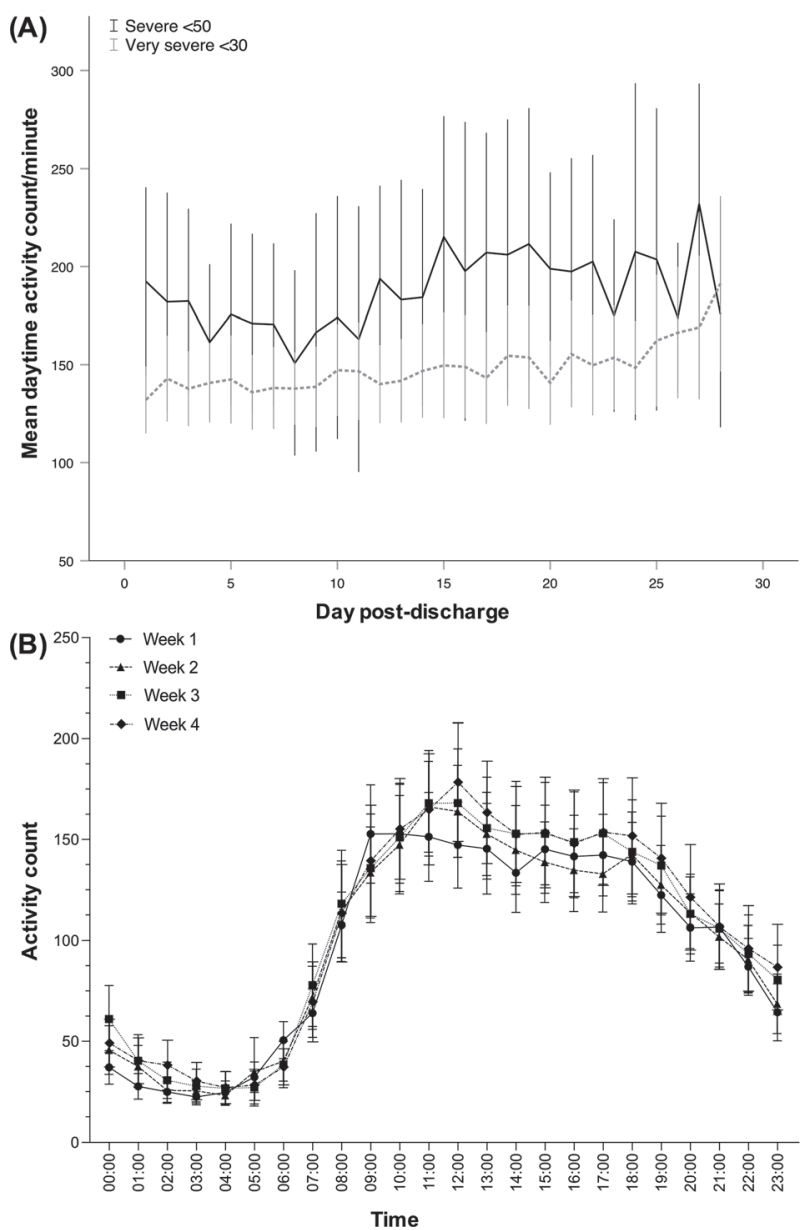

Abstract S29 Figure 1 (A) Daily physical activity in patients with severe $(n=15)$ or very severe $(n=48)$ airflow obstruction, (b) Hourly physical activity count per 24-hour period for 4 weeks post-discharge following sentence AECOPD. 